\title{
ESTUDIO ARQUEOLÓGICO Y URBANÍSTICO DE LA “CASA DE LA REINA", UNA VIVIENDA ECLÉCTICA (1859) DEL BARRIO MARÍTIMO DE EL CABA- NAL DE VALENCIA
}

\author{
Víctor M. Algarra Pardo ${ }^{1}$
}

\section{Resumen:}

El estudio arqueológico de la Casa de la Reina, un edificio de mediados del siglo XIX situado en el barrio marítimo del Cabañal de la ciudad de Valencia, nos ha permitido, en primer lugar, llevar a cabo una aproximación al particular urbanismo de Pueblo Nuevo del Mar, municipio independiente desde el año 1837. Una trama urbana que copia los modelos de ensanche de las principales ciudades españolas de este mismo periodo, con un trazado de calles ortogonales paralelas a la línea de playa. Asimismo, el análisis arqueológico de las estructuras del edificio definió la evolución arquitectónica del mismo y el reconocimiento de la distribución y funcionalidad de los espacios.

\section{Palabras clave:}

Siglo XIX, arqueología contemporánea, urbanismo, El Cabañal, arqueología de la arquitectura.

\begin{abstract}
:
The archaeological study of Casa de la Reina, a building from mid XIX century located in the maritime neighbourhood of El Cabañal in Valencia, has allowed us, in the first place, to conduct an approximation to the particular urbanism of which it was an independent municipality since 1837. An urban web that copies the models of the growths of the main Spanish cities from the same time, with orthogonal streets parallels to the line of coast. The archaeological analysis of the building's structures defined the architectonical evolution of it and the acknowledgement of the distribution and functionality of the spaces.
\end{abstract}

\section{Keywords:}

XIX Century, contemporary archaeology, urbanism, El Cabañal, archaeology of architecture.

1 Gabinete de Arqueología Algarra y Berrocal. La Linde, Revista Digital de Arqueología Profesional - lalindearqueologia@gmail.com 


\section{Introducción}

En el año 1837 los actuales barrios de El Cabañal, Cañamelar y Cabo de Francia, que forman la fachada litoral de la ciudad de Valencia al norte del puerto, obtuvieron la independencia municipal bajo el Ayuntamiento de Pueblo Nuevo del Mar. Este hecho impulsó una necesitada ordenación urbana del nuevo municipio, constituido hasta el momento por barracas de pescadores. En 1840 se planificó un singular urbanismo formado por manzanas paralelas a la playa, siguiendo un trazado ortogonal.

En uno de los solares reparcelados en 1840, tras una serie de compraventas en ocasiones puramente especulativas, Bernabé Dombón y Olivar, vecino de la Villa y Corte de Madrid, constructor de máquinas de Su Majestad, construyó entre 1859 y 1862 su residencia y también taller para sus inventos, entre ellos una máquina voladora, a cuya presentación deseaba invitar a la Reina Isabel II, inicio de la leyenda que bautizó a la vivienda como "Casa de la Reina" (la máquina fue un fracaso y se estrelló en la playa de la Malvarrosa sin la asistencia de la Reina).

El estudio de Arqueología de la Arquitectura que pudimos realizar en este edificio, actual $n^{\circ} 85$ de la calle de la Reina, permitió perfilar su evolución arquitectónica, determinar los materiales y técnicas constructivas, registrar sus numerosos elementos suntuarios y reconocer la distribución y funcionalidad de sus estancias, tanto las de vivienda, como las de taller.

El análisis de este edificio eclético sirve, además, para comprender en su justa medida los valores urbanísticos del barrio valenciano de El Cabañal, que cuenta con una calificación de Bien de Interés Cultural, sometido a una larga polémica y movilización ciudadana ante la proyectada "reforma" de este barrio marítimo.

\section{La planificación urbanística de la calle de la Reina en el municipio de Pueblo Nuevo del Mar}

La ciudad de Valencia y su entorno, lo que se conoce actualmente como la comarca de I'Horta, inicia el siglo XIX todavía en el seno de la organización territorial y fiscal de origen bajomedieval conocida como Particular Contribución, basada en la tradición jusnaturalista del Antiguo Régimen, que consideraba el municipio como un ente natural al que se asociaban otras entidades de régimen señorial civil o religioso y de realengo. La Valencia envuelta por sus viejas muraIlas, como centro de la Particular Contribución, ejercía de facto un sometimiento fiscal a un territorio que, por otro lado, constituía una extensa área histórica de huerta totalmente interrelacionada (fig. 1). En un primer radio de menos de $5 \mathrm{~km}$ de la capital se desplegaba un hábitat diseminado pero denso en alquerías y núcleos de población mayores, conocidos como Llocs, que en la actualidad son to- 
dos ellos barrios consolidados de Valencia. Superado este primer cinturón seguía un modelo semejante con otros /locs hoy también incluidos en la capital, pero la mayoría municipios independientes en el Área Metropolitana de Valencia.

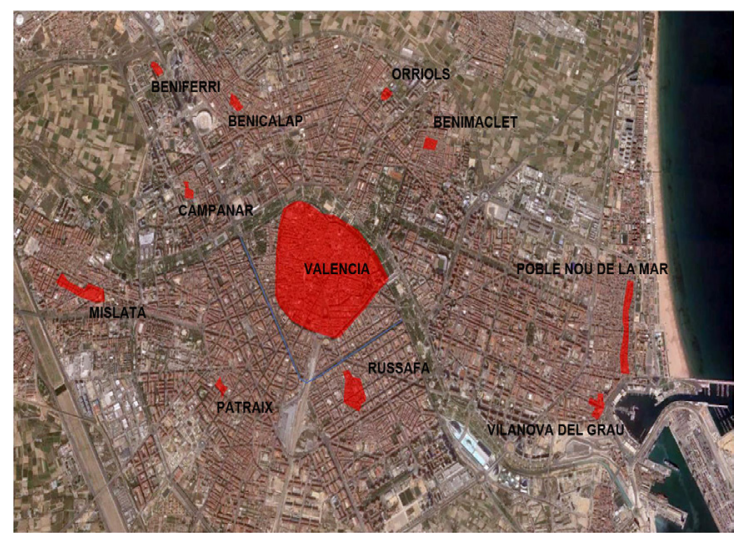

Fig.1. Localización en la fotografía aérea actual de Valencia de los antiguos pueblos (hoy barrios) de la Particular Contribución que formaban parte del área más próxima de la ciudad.

Pero tras la Guerra de la Independencia los días del Antiguo Régimen estaban contados y su disolución representó en el ámbito local la generalización de un nuevo orden municipal, nacido de la Constitución de Cádiz. Los antiguos Llocs o pueblos de la Particular Contribución comienzan a demandar la autonomía municipal desde 1813, el pronunciamiento de Riego en 1820 animó nuevas segregaciones y finalmente a partir de 1836 la totalidad de las poblaciones de la Particular Contribución se constituyeron en municipios independientes (incluso algunos arrabales extramuros de la ciudad también solicitaron la segregación), lo que de manera inmediata fomenta un impulso reformador en sus antiguos cascos urbanos.

El Cabanyal, Canyamelar y Cap de França ${ }^{2}$, las tres áreas que forman la fachada marítima al norte del Grao, constituyeron uno de estos municipios independientes en el periodo comprendido entre los años 1837 y 1897, recibiendo el nombre de Pueblo Nuevo de la Mar (Poble Nou de la Mar). Su origen es el de una serie de viviendas más o menos marginales de pescadores, que se asentaron en barracas fuera del núcleo amurallado de la Vilanova del Grau (el puerto de Valencia), en una partida que ya se conocía como el "Cabanyal" en la documentación de principios del s. XV (Sanchis, 1997: 16). El Cabanyal fue consolidándose como un núcleo de pescadores, pero también de agricultores, en el territorio de

2 El libro de A. SANCHIS PALLARÉS: Historia del Cabanyal. Poble Nou de la Mar (1238-1897). Javier Boronat, editor, Valencia: 1997, es una documentadísima obra que recoge la historia de este antiguo municipio. 
huerta regado por el final del sistema hidráulico de la gran acequia de MestaIla. A finales del siglo XVIII existían allí más de doscientas barracas, las cuales sufrieron un incendio devastador en el año 1796 que consumió la mayor parte de ellas, por tratarse de construcciones realizadas con materiales tan fácilmente inflamables como son las cañas y la paja. Este incidente será el primer indicador de la necesidad de contar con unas normas urbanísticas en materia de ordenación del espacio y tipo de edificaciones con materiales de construcción más seguros como por ejemplo el ladrillo.

La construcción del nuevo muelle del puerto en 1792 implicó el cambio en la dirección de los oleajes de manera que también se modificó la sedimentación de la arena de la playa, propiciando una retirada del mar y la formación de nuevas tierras por el cambio de la línea de playa. Desde los primeros años del s. XIX existe un expreso deseo de urbanizar esta área, que se materializará tras la obtención en el año 1837 de la independencia municipal bajo el Ayuntamiento de Pueblo Nuevo del Mar, definiéndose las líneas fundamentales del moderno urbanismo del Cabañal, que han Ilegado hasta nuestros días, con eje principal en la calle de la Reina.

En ello influyó de manera especial la costumbre que, desde finales del s. XVIII pero sobre todo ya entrados en el XIX, se instauró entre las clases más pudientes no sólo de la ciudad del Valencia sino también del resto de España, en particular de Madrid, de pasar una temporada del año junto a la playa para tomar los curativos baños de mar. La burguesía de esa época comenzó a interesarse por la posesión de una vivienda de veraneo junto al mar y sin duda el Cabañal reunía las condiciones más propicias: su cercanía a la capital, las facilidades que el nuevo municipio presentó para su asentamiento conocedor de los beneficios que ello le reportaría, las características de su playa arenosa y la existencia de una importante extensión de terreno sin urbanizar y sin propiedad, a parte del propio Estado representado por la Bailía General.

\section{El proyecto de parcelación de la calle de la Reina y la formación del solar no 85}

En el año de 1840 el Ayuntamiento de Pueblo Nuevo del Mar emprende junto con la Bailía General del Real Patrimonio un plan de urbanización del área comprendida entre las manzanas que dan a las calles de la Reina y calle Barracas y, por otro, a la calle de la Reina y a la calle del Doctor Lluch, comenzando por el sur desde la actual calle de Francisco Cubells hasta la avenida del Mediterráneo por el Norte, que vienen a sumarse a las del núcleo originario asentado al oeste, algo más separado de la línea de costa, en calles como las de Alegría, Rosario, Escalante, José Benlliure o Los Ángeles (Pastor, 2012). 
Esta parcelación se plasmó en un plano levantado por el arquitecto José Serrano $^{3}$ (fig. 2A). Se diseñan en total 12 islas de casas, 6 al este de la c/ de la Reina y otras tantas al oeste, que acogían a 124 parcelas, cuyo eje central iba a ser la calle de la Reina. Las dimensiones de las parcelas fueron siempre las mismas, con un ancho de fachada de 30 palmos valencianos, es decir $6.79 \mathrm{~m}$, y una profundidad de 90 palmos (20.38 m). Las parcelas 122 y 120 son el origen de la Casa de la Reina en el actual número 85 de la calle, adjudicadas en primera instancia a Bautista Marí la n 122 y a Pascual Hernández la nº 120.

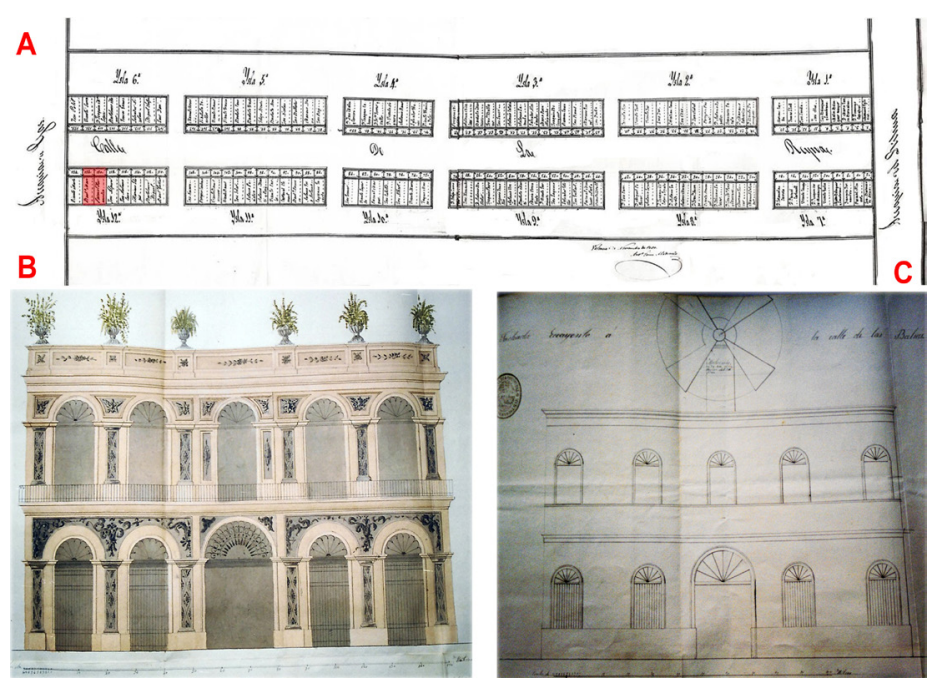

Fig. 2. A. Proyecto de parcelación de la Calle de la Reina. 1840. B. Alzado de la fachada de la Casa de la Reina. Proyecto arquitectónico de Joaquín Cabrera. 1861. C. Alzado de la fachada del taller de la calle Barraca. Proyecto arquitectónico de Joaquín Cabrera. 1861.

La concesión de la parcela se formalizaba mediante un documento de establecimiento y perpetua enfiteusis ante el Baile General, en el que se marcaban una serie de cláusulas de obligatorio cumplimiento a la hora de la construcción de las viviendas y el plazo de la edificación, transcurrido el cual si no existía construcción se perdía la titularidad. Como veremos posteriormente este plazo fue muy laxo, concediéndose muchas prórrogas.

Se han conservado en el Archivo del Reino de Valencia (ARV) los documentos de los años 1840 y 1841 mediante los cuales el Baile General de la ciudad de Valencia recoge las solicitudes e instruye expediente sobre las demarcaciones de la parcelas ${ }^{4}$. Nos fijaremos como ejemplo en las cláusulas de la parcela 122 de Bautista Marí y sucesores. En primer lugar se señala su concreta localización

3 Se conserva copia de este plano en ARV Bailia, letra B, leg. 13, exp. 167. 4 ARV, año 1840, Bailia, letra B, Leg. 16. 
dentro de la parcelación de la calle de la Reina, así como sus dimensiones: "patio solar número 122 de la Isla duodécima de la espresada calle de la Reyna, partida del Cañamelar, comprensivo de 32 palmos de ancho, y 90 de fondo, lindante por un lado con el número 124, señalado a Vicente Martí, y por el otro con el número 120 señalado a Pascual Hernández bajo las condiciones siguientes..."

Siguen otras cláusulas como la obligación del enfiteuta Bautista Marí de pagar cada año 10 sueldos por canon a la Bailía, la cual retenía el dominio mayor y directo, o como la que se dispone que en el plazo de un año a partir de la fecha del 27 de abril siguiente, si no se había edificado casa alguna, el documento quedaba nulo, no teniendo el concesionario derecho sobre el solar. La Bailía se reservaba el derecho de cedérselo a otro solicitante.

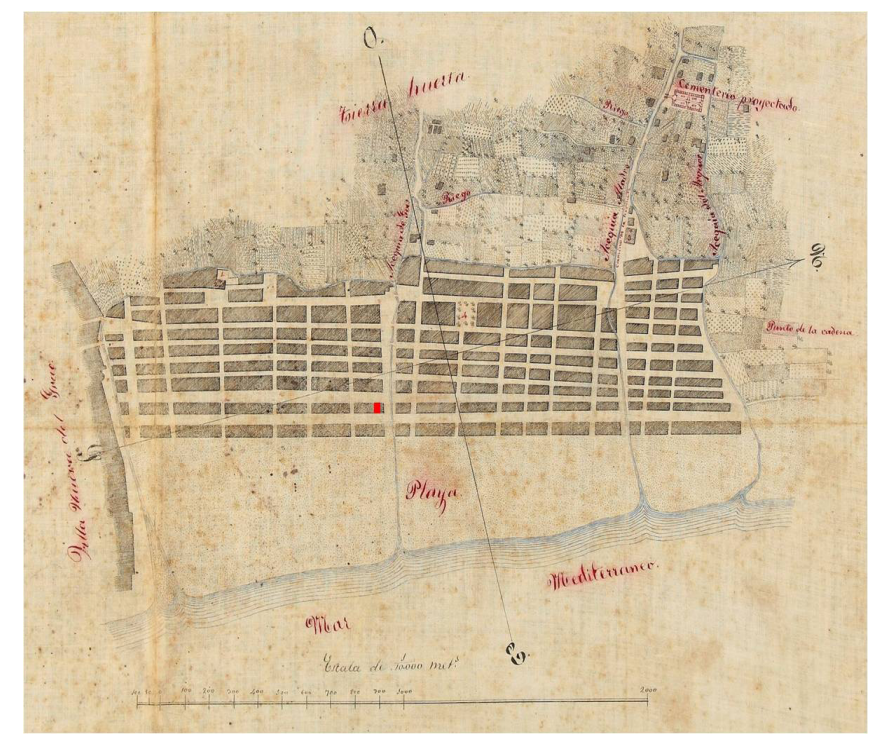

Fig. 3. Situación de la Casa de la Reina en el Plano de Pueblo Nuevo del Mar. 1860. Archivo Diputación Provincial de Valencia. MP. 27, nº14.1.

En otras se establece que los edificios de la parte del Mar, es decir los que en la actualidad dan a las calles de la Reina y Doctor Lluch, sean "casas bajas al piso de tierra", sin que se pueda levantar otro piso en el interior y "en lo esterior tampoco más que la fachada principal que mira a la calle...". Sin embargo, las manzanas que dan al interior, es decir las que dan a la calle de la Reina y de San Antonio, la actual calle Barracas "serán casas al piso de tierra con habitación alta". De esta manera los solares 120 y 122 se hallaban en el grupo de futuras viviendas con posibilidad de construir un piso sobre la planta baja.

Otras cláusulas aluden a medidas de homogeneización de fachadas y entorno urbano, como la sexta en la que se expone que "... también es condición que 
todos los edificios ó casas de la mencionada calle, han de tener un anden comprensivo de todo lo ancho de su fachada principal, y lo mismo la de las espaldas de doce palmos valencianos de ancho $[2,72 \mathrm{~m}]$, unidos unos con otros entre ambas casas sin que suban ó bajen unas mas que otras, debiendo todas estar perfectamente alineadas; á las que se les marcará la altura y nivel que deberán tener respecto á la superficie de la calle, cuya construcción será formando una pared del espesor ó grueso de un ladrillo que podrá ser tambien de mamposteria ordinaria: su coronación en cualquier clase de losas de sillería y su terraplen de piedra y graba buena de Rio".

A partir de este núcleo diseñado en 1840 fue materializándose en sucesivos planeamientos un peculiar urbanismo de manzanas rectas y paralelas a la línea de playa, donde se conjugan sectores con viviendas más o menos suntuosas, como la Casa de la Reina, situada en el $n^{\circ} 85$ de la c/ de la Reina, con otras de predominio de barracas. Un urbanismo que sigue los principios de la ortogonalidad típica de los ensanches de la segunda mitad del siglo XIX. En el plano de $1860^{5}$ de Pueblo Nuevo del Mar se ha alcanzado la máxima expansión del planeamiento del municipio, contenido al sur por el límite con el municipio de la Vilanova del Grao y por el norte la acequia del Arquet o de la Cadena en una cuadrícula de calles rectas, que, salvando la dimensión del territorio, se asemeja a las soluciones adoptadas hacia las mismas fechas en grandes ciudades españolas incluida la propia ciudad de Valencia (fig. 3).

\section{Evolución de la propiedad del solar y construcción del taller con fachada a la calle de la Barraca}

Entre 1840, fecha de la planificación de las parcelas, y el inicio de las primeras obras en el solar de la futura Casa de La Reina pasan más de 17 años sucediéndose ventas del solar, decomisos por no construir en los plazos establecidos y prórrogas de construcción, algo común en otras muchas parcelas ${ }^{6}$. Finalmente entre 1857 y 1858 Juan de Dios Lamban, propietario desde 1854, construye el cerramiento del solar hasta una altura de 3,17 m. Pero finalmente se vendió en 1859 a Bernabé Dombón Olivar ${ }^{7}$, tras 2 operaciones especulativas, pues en 10 meses el precio del solar pasó de 6000 reales de vellón a 13.000 y posteriormente a 19.500 reales. Bernabé Dombón Olivar terminó la edificación, no exenta de pa-

5 Plano de Pueblo Nuevo del Mar. 1860. Archivo Diputación Provincial de Valencia. MP. 27, n¹4.1 Diputación 8035.

6 En 1854 todavía restaban por edificar un total de 53 solares de los 124 iniciales, 2 sólo tenían cimientos y 7 estaban en construcción.

6 ARV. Protocolo, 9854 de Timoteo Liern, año 1859, registro 195 de 30 de marzo de 1859. Venta de las parcelas 120 y 122 de la calle de la Reina por parte de Amalia Navarro Selma a Bernabé Dombon Olivar. 
ralizaciones cautelares por carecer de permisos y por instalar ilegalmente ciertos artilugios, que implicaban la construcción de una vivienda con entrada a la calle de la Reina y otro edificio a sus espaldas con función de taller, respondiendo al oficio de inventor del dueño de la casa.

Bernabé Dombón, inventor de máquinas de Su Majestad, fue un personaje peculiar que como ha perdurado en la tradición oral y por los documentos consultados debió caracterizarse por un carácter resolutivo y emprendedor. Nacido en Zaragoza hacia $1812^{8}$ con residencia a caballo entre Valencia y Madrid, debía poseer una renta importante como testimonia el precio que pagó por el solar, 19.500 reales, y el desembolso inmediato que significó la edificación de una parcela el doble de grande que la mayor parte de las parcelas del barrio.

Fue él mismo quien dirigió las obras al comienzo de la edificación, razón por la cual fue amonestado y se le requirió la presencia de un arquitecto, Joaquín Cabrera, quien firmará los planos de un proyecto que para entonces ya estaba ejecutándose y posiblemente en un estado muy avanzado 9 .

Utilizó la casa, además del uso lógico de residencia, como lugar donde realizar una serie de inventos ${ }^{10}$. El más conocido fue el de la Máquina Dombon, como el mismo la llamaba, un aparato volador que recibió no pocas críticas y mofas $^{11}$. Rafael Solaz (2014) recoge algunos datos de este invento en forma de pájaro a modo de ala delta, con el que pretendía volar en 1864 pasando por el Palacio Real de Madrid y seguir en dirección a Filipinas: el "pájaro-buque tenía las dimensiones de un falucho (especie de lancha), un camarote de proa con dos butacas y otro camarote en la popa, el destinado al director o capitán. Llevaba una rueda de hélices y era casi todo de hierro". Éste como otros de sus inventos cuentan que los fabricaba en la parte de la casa recayente a la calle Barraca, siendo por ello su taller. La funcionalidad de esta parte del edificio como nave en la que pueden practicarse actividades diversas ha quedado demostrada en el estudio arqueológico.

La máquina voladora resultó funesta para don Bernabé pues parece ser que el invento fue un fracaso y tema de escarnio entre sus vecinos. Este motivo pudo influir en la venta de la casa a José de la Cruz Baya en 1868.

8 Datos obtenidos por una citación judicial anotada en el Diario Oficial de Avisos de Madrid con fecha de 12 de enero de 1870 http://hemerotecadigital.bne.es/pdf.raw?query=id:0000395708\&lan$\mathrm{g}=\mathrm{es} \& \log =00000000-00000-00001 /$

9 A.H.M. Pueblos anexionados. Pueblo Nuevo del Mar, caja 10/60. Expediente de licencia de obras del edificio de la calle de la Reina 117-119. 1860.

10 De 1847se conoce una mención de su oficio de inventor de máquinas cuando obtuvo un premio de la Real Sociedad Económica de Amigos del País de Valencia por una máquina hidráulica para elevar agua. RESEAP 1847, C-118, II Industria y Artes n8, doc. 3280.

11 En la edición del 12 de mayo de 1858 de La lberia, diario liberal de la mañana, publicado en Madrid, Bernabé Dombon redacta una nota quejándose de la incomprensión y sátira recibida por la invención de su Máquina Dombon. 
Volviendo a las circunstancias que se recogen en el expediente de licencia de obra de la casa, el 8 de junio de 1860 el Arquitecto de la provincia, Antonio Sancho, remite al alcalde de Pueblo Nuevo del Mar un informe en el que se manifiesta que se está construyendo un edificio propiedad de Bernabé Dombón sin licencia y sin la dirección de un arquitecto o un maestro de obras, lo cual contraviene la ordenanza. De su contenido se desprenden dos datos, el primero que las obras se ejecutan bajo la propia dirección de Bernabé Dombón, el cual es calificado como constructor de máquinas de Su Majestad y, como tal, él se considera facultado para dirigir la edificación, y la segunda que el edificio tiene por destino un establecimiento de construcción de máquinas. El resultado es el dictamen de paralización de obras, imposición de multa y redacción de un proyecto por un arquitecto facultado.

Pero Bernabé Dombón ni paralizó la obra ni pagó la multa. Es más, el 2 de marzo del año siguiente, 1861, el alguacil Ramón Rodes comunicó al alcalde de Pueblo Nuevo del Mar que en la mañana de este dia habia observado que en el edificio de la calle de la Reyna de la propiedad de Don Bernabe Dombon y cuya obra estaba en suspenso por orden del Sr. Gobernador se habia elevado en la parte posterior recayente a la calle de San Antonio una chimenea de hierro para servir de motor a las maquinas que el Dombon habia colocado en aquella parte del edificio. A pesar de estas denuncias desatendidas, al mes siguiente remite planos debidamente firmados por un arquitecto y de esa manera se emite el definitivo informe de licencia.

En ese informe de inspección final encontraremos más datos acerca de ese extraño artilugio que sobresalía por la fachada trasera y que sin duda debió ser muy comentado por el vecindario: en el taller que había construido para realizar allí la maquinaria de su invención existían diferentes piezas de maquinaria que sirven para tornear, [----], hacer roscas, etc, etc., diferentes objetos de hierro forjado y fundido sin que exista ni horno de fundición ni calderas de vapor, pues en la actualidad no hay otro motor que un [----] servido de caballerias y dos forjas de las comunes y que por su aislamiento no ofrecen cuidado alguno, para caldear las piezas de hierro que hayan de trabajarse.

Respecto a la chimenea de la que se le exigió su derribo se especifica que en realidad se trataba de una columna de hierro elevada en lo interior y parte céntrica del establecimiento y que se ha juzgado por la generalidad de los que la han visto destinada a servir de chimenea, tiene por objeto apear el aparato y aspas colocado en la parte superior de la misma para utilizar el viento como motor de la maquinaria del taller en lugar del [---] que hoy existe. El juego de engranaje combinado de alto a bajo por medio de las correspondientes varillas o palancas se verifica por la parte interior de dicha columna, cuyo vacio interior tiene aproximadamente un diametro de un metro. La columna insiste sobre un cimiento solido de mamposteria coronado por silleria trabada y enlazada convenientemente para evitar la so- 
lución de continuidad de modo que segun he podido observar parece trabajando [-----] conforme a las [------] del Arte de Edificar. Además de esto se halla sujeta dicha columna en diferentes puntos de su altura a fin de evitar cualquier pequeño desplome que redundaria en perjuicio de la regularidad del movimiento que necesitan los aparatos de la maquinaria para funcionar cual corresponde.

Contamos con el croquis de la columna de hierro y las aspas que se elevan en el alzado de fachada trasera adjuntado al informe (fig.2C) donde se incluye la leyenda motor colocado en el interior del edificio ${ }^{12}$.

El estudio arqueológico de la nave de la calle de la Barraca permitió reconocer huellas de esta antigua instalación. A pesar de que la vivienda de la calle de la Reina 85 y el edificio con el número 88 de la calle de Barraca han formado parte desde el momento de su construcción de una misma propiedad y, aun existiendo puntos originales de comunicación entre ambos, estructuralmente podemos considerar que los dos inmuebles son independientes por su sistema murario, por las cotas de sus forjados y por el sistema de cubiertas. Entre ambos existe un espacio de transición representado por el patio flanqueado a cada lado por sendas habitaciones de una sola planta con terraza, de manera que todavía se imprime una mayor autonomía a cada uno de los dos grandes bloques.

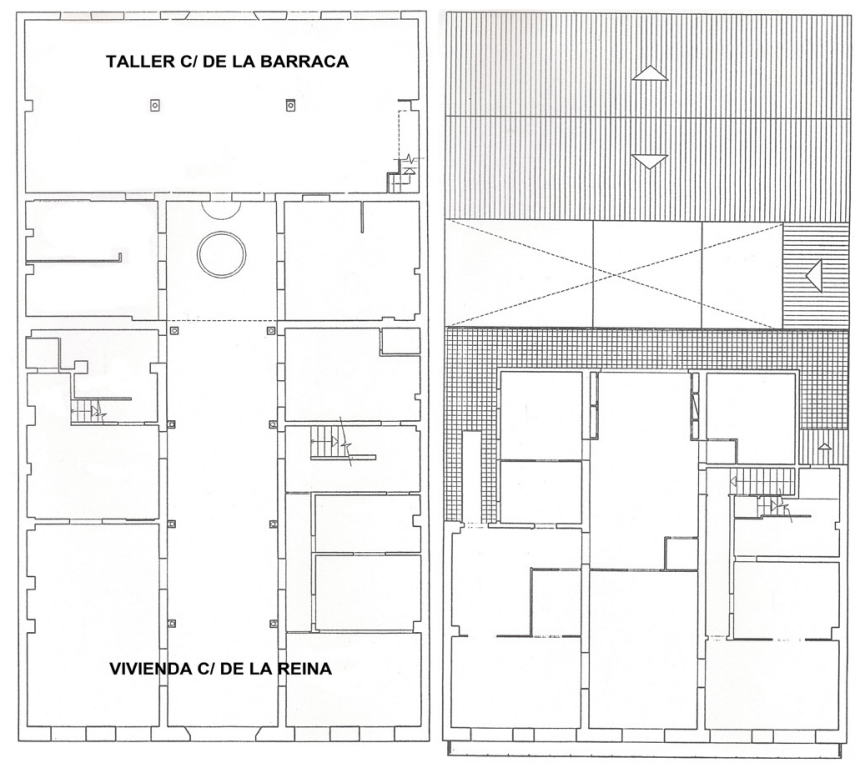

Fig. 4. Planta baja (izquierda) y planta superior (derecha) de la Casa de la Reina.

12 En un grabado de la época, conservado en la colección privada de Rafael Solaz se puede apreciar este singular molino de viento que se proyecto sobre el tejado de lo que sin duda es la Casa de La Reina del Cabañal, puede observarse en http://vlcnoticias.com/dombon-un-pionero-dela-aviacion-espanola-vecino-de-la-calle-de-la-reina-en-el-cabanyal/ . 
La documentación de archivo nos ha permitido conocer que el grueso de la construcción se hizo entre marzo de 1859 y junio de 1860 y que desde esa última fecha hasta la de marzo de 1861 la obra estuvo paralizada cautelarmente, finalizándose entonces con la instalación definitiva del molino de viento.

El taller de la calle Barraca (fig. 4), presenta una planta rectangular de unos $15.20 \mathrm{~m}$ de longitud por $7.50 \mathrm{~m}$ de profundidad, con dos crujías paralelas a fachada de idéntica anchura interna: $3.30 \mathrm{~m}$. Posee dos niveles, planta baja y cambra o piso superior, y la cubierta es a dos aguas vertiendo a la calle y al patio interior respectivamente. El estado con el que llegó a la actualidad reflejaba modificaciones en la estructura original por los nuevos usos recibidos tras la venta del inmueble a Juan de la Cruz Baya en 1868, que lo arrendó, siendo utilizado en diversos momentos como cuadra para los caballos del Tranvía de Pueblo Nuevo del Mar y también como lechería.

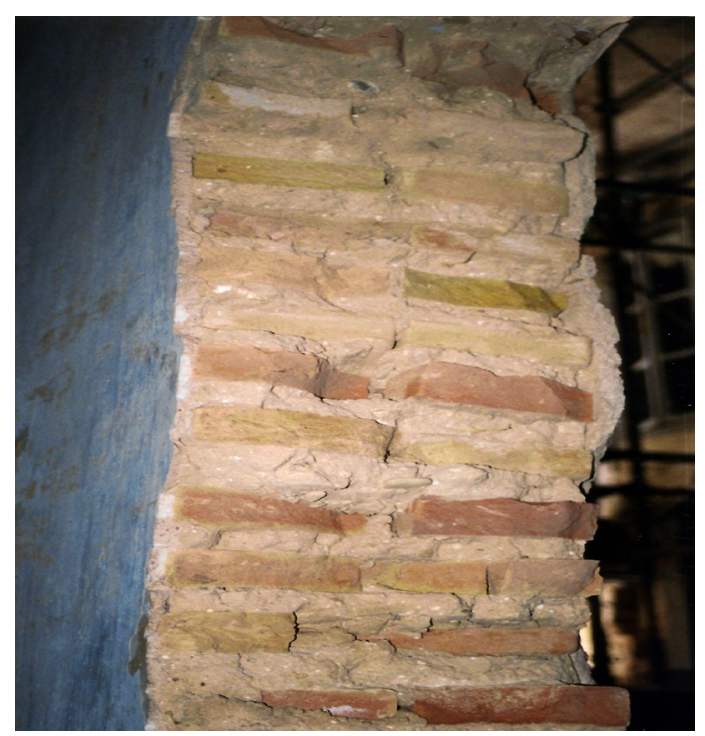

Fig. 5. Ejemplo de muro de obra de ladrillo.

En el momento de su construcción estaba directamente vinculado con el edificio principal mediante un sistema de vanos que daban al patio y a las dos habitaciones contiguas a éste. El interior era totalmente diáfano, sin la tabiquería que posteriormente se realizó. Los muros del taller y los de la vivienda son de obra de ladrillo, con un ancho aproximado de $35 \mathrm{~cm}$. Se realizan con ladrillos de $28 \mathrm{~cm}$ de longitud, medida ésta que ratifica la cronología de comienzos de la segunda mitad del siglo XIX para el momento de construcción del taller (fig. 5). El trabado se realiza con mortero muy compactado de arena de textura arcillo-limosa con gránulos de cal. Las hiladas están muy regularizadas con tendeles de $3.5 \mathrm{~cm}$ y llagas de $0.5 \mathrm{~cm}$. La cimentación 
consiste en una banqueta de mampostería, coronada por una capa de yeso que baja en bisel unos $15 \mathrm{~cm}$. El nivel de suelo original de la planta baja se encontraba a 40 cm del pavimento actual. Se componía de baldosas de pasta roja de $34 \mathrm{~cm}$ de lado, dispuestas a juntas encontradas sobre una preparación de arena de playa.

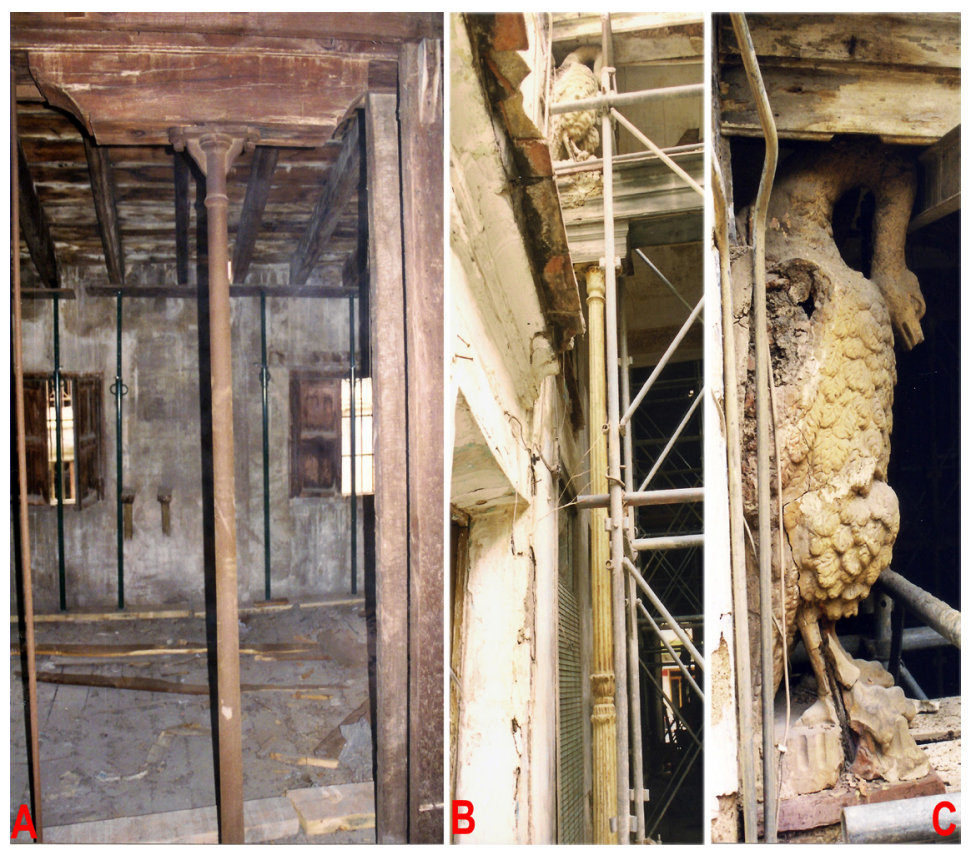

Fig. 6. A. Columna de hierro fundido del cuerpo de taller en la calle de la Barraca. B. Columna de hierro fundido y remate superior de ave de terracota del cuerpo central o pasillo de la planta baja de la Casa de la Reina. C. Detalle del ave de terracota.

La separación de las dos crujías se realizaba originalmente en planta baja mediante sendos pilares de obra en los extremos Norte y Sur de la nave y dos columnas de fundición. Los pilares tienen una forma en la parte superior de medio trapecio. Las columnas cilíndricas, situadas equidistantes entre ellas y los muros de los extremos Norte y Sur, poseen una base cimentada de piedra, rematándose con una pieza oval sobre la que descansa una pieza de madera con moldura de caveto derecho que se interpone entre el capitel y la viga maestra (fig. 6A).

El forjado se sustenta sobre la viga maestra y sendas carreras que descansan en los muros largos y en ménsulas o canecillos de ladrillo escalonados. La viga maestra está cajeada para ampliar su sección, de manera que pueda poseer el ancho del tabique de madera de la cambra. El forjado se complementa con viguetas de unos 12-15 cm (algunas con un rebaje moldurado en los ángulos y otras de mayor sección y sin acabado final, denotando una posible reforma del forjado), sobre las que se disponen las tablas de madera del suelo. 
La cubierta también se realiza mediante viguetas de madera y entarimado de listones de madera al interior, cubriéndose al exterior con teja árabe. La sustentación de la viga maestra repite el sistema de la planta baja, mediante el uso de pilares de obra en los extremos y columnas de fundición.

A la planta superior se accedía a través de una escalera actualmente anulada, situada en el extremo Noreste del taller. Las dos crujías de la nave estaban divididas mediante un original tabique de madera en el que se dejaron una serie de vanos, unos con paso franco y otros en los que se interponían las columnas de fundición que sostienen la cubierta (fig. 7). El tabique se componía de una serie de estacas asentadas en la viga maestra del forjado, emparejadas dos a dos y unidas mediante listones horizontales. Los paramentos se cerraban con tablones a modo de faldones. De éstos tan sólo han quedado unos pocos restos en el extremo Sur. De esta manera el tabique poseía una cámara interna, únicamente interrumpida por varillas metálicas situadas en el interior que de tanto en tanto ataban la viga maestra del forjado y la cumbrera de la cubierta respectivamente.

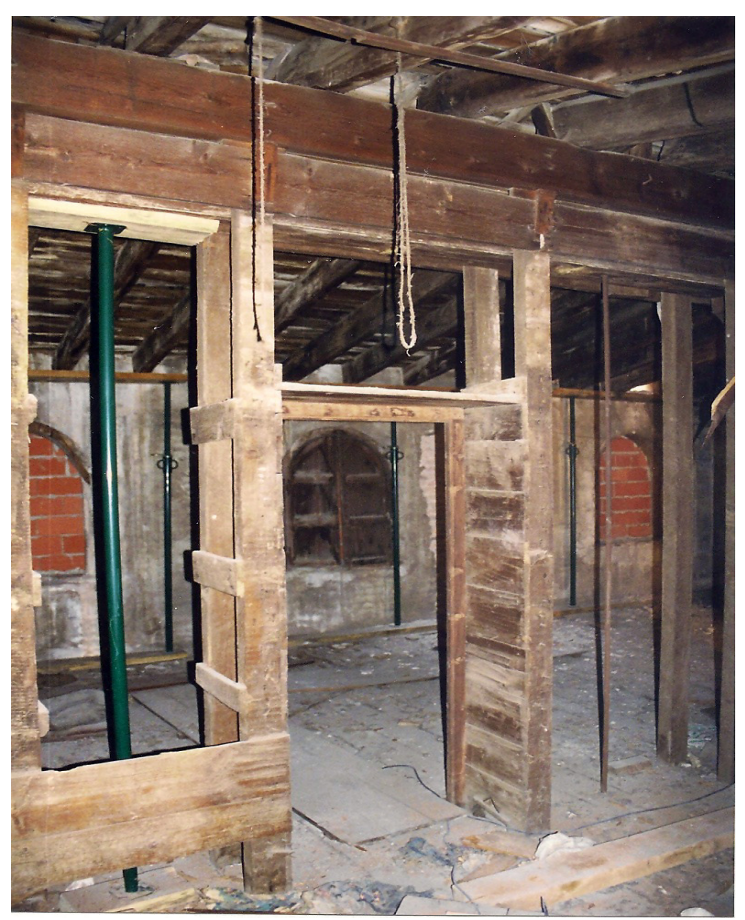

Fig. 7. Detalle del tabique de madera y puerta entre crujías del taller de la C/ Barraca.

En el centro de la nave, y cerrado también en origen por tablones, se hallaba una viga de mayor calibre que las que formaban la estructura del tabique, la cual se ha relacionado con el eje o árbol del artefacto llamado en la documentación 
"chimenea", perteneciente al motor de viento instalado por Bernabé Dombón, incluso se conservaba un rebaje semicircular con signos de rozamiento en esta viga (Lám. 8). El aire fue la fuerza motriz de la maquinaria del taller, transmitida a través de ese árbol o eje a un embarrado con poleas y correas, artefacto poco habitual en tierras valencianas, en especial en el entorno de la huerta de Valencia, donde el agua fue la fuente tradicional de energía, sustituida en el siglo XIX por el vapor y más tarde por el gras pobre y la electricidad. Finalmente, relacionado también con el taller, en el muro trasero perduraban los restos de una estructura de madera consistente en una serie de caballetes empotrados que formarían parte de una bancada de trabajo de este taller.

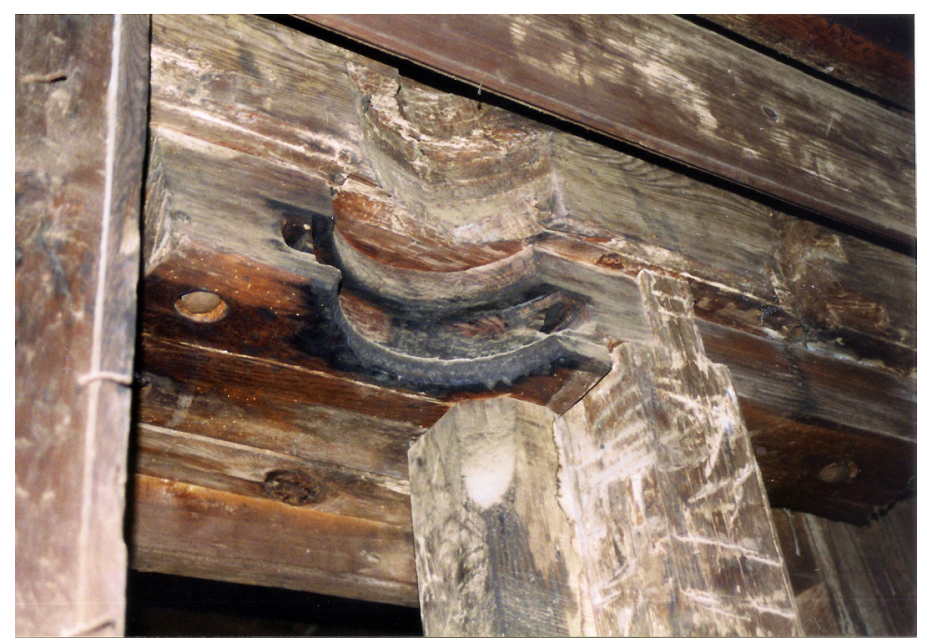

Fig. 8. Detalle de la muesca para alojar el árbol o eje del molino de viento.

\section{Análisis de la vivienda de la calle de la Reina: del proyecto original a la plasmación definitiva}

\section{Descripción de la fachada}

Pasando a la parte de vivienda con acceso desde la calle de la Reina, el diseño original de su fachada (fig.2B), que podemos observar en el alzado del proyecto dibujado por el arquitecto Joaquín Cabrera (hijo del que fue arquitecto mayor del Ayuntamiento de Valencia también Ilamado Joaquín Cabrera), respetó en esencia la resolución final de la obra en cuanto a la disposición de los vanos y su morfología, la división de plantas con su molduraje y el tipo de balcón y de antepecho. Las diferencias se encuentran sobre todo en los motivos decorativos de las enjutas y pilastras.

Esta fachada se incluye en el estilo arquitectónico conocido como historicismo romántico y más en concreto el estilo isabelino o clasicismo isabelino, generalizado a partir del segundo tercio del siglo XIX en España. Muy brevemente diremos 
que con el final del clasicismo y por la influencia del pensamiento romántico que invade las ideas estéticas, las artes, y en concreto la arquitectura, mostrarán un programa de corte historicista. Éste será entendido por algunos como un revival del pasado, fundamentalmente el bajomedieval, con una concepción pretendidamente arqueologista (aunque en ningún momento de manera pura), y por la mayoría contemplado desde un punto de vista ecléctico. Es decir, se retomaron estilos del pasado, en muchas ocasiones mezclándolos, para alcanzar uno nuevo, el cual se pretendía que fuera el definitorio de la sociedad burguesa.

La vivienda de la calle de la Reina se encuadra en uno de estos eclecticismos emergentes, el del neorrenacimiento, el cual, a pesar de distanciarse del monolítico clasicismo que se había mantenido en el siglo XVIII y el primer tercio del XIX como el estilo único, deberá considerarse aún así como un clasicismo renovado que ya no se basará tanto en el Mundo Antiguo grecorromano como en la vertiente también clasicista del Renacimiento, de ahí la calificación de clasicismo isabelino.

Fue el del neorrenacimiento uno de los eclecticismos con menor número de obras en España, aunque con ejemplos tan notables como el del Palacio del Marqués de Salamanca en Madrid, del que encontramos ciertos paralelismos en la casa de la calle de la Reina, siempre muy relativizados y guardando las distancias en cuanto a volumen, suntuosidad y programa arquitectónico.

Es en la fachada donde se sintetizan las características de esta arquitectura. El proyecto de fachada principal contemplaba su división en dos niveles, el primero abarcaba la planta baja y el entresuelo y el segundo el piso principal, divididos ambos por un balcón corrido. En ambos niveles existía un juego de arcadas con arcos de medio punto, al modo de la fachada del citado Palacio del Marqués de Salamanca, con un total de cinco vanos por planta. Estos arcos no funcionan en realidad como tales, pues presentan un friso decorado a la altura de las impostas al exterior y un dintel al interior que transforma al vano en adintelado. De esta manera el espacio del arco propiamente dicho forma una serie de tímpanos que en el proyecto original estaban enrejados en ambos niveles y en la ejecución final se ciegan y decoran con relieves en el nivel superior.

El nivel inferior poseía el arco central más ancho, correspondiendo éste con el acceso principal del pasaje o eje central que articula el interior de la vivienda. Entre los arcos se disponían pilastras adosadas sobre una base aparentemente de sillería. Los dos pares de arcos a los lados del central debían ser ventanas y no puertas, a pesar de que el vano parte desde el suelo mismo. De cualquier modo, en el proyecto queda perfectamente indicado que los vanos no permitían el paso pues éstos estaban cerrados por una reja desde la imposta de los arcos hasta la base. El arco propiamente dicho también estaba cerrado por una rejería de pétalos o gajos de naranja, a los que se añaden en el central otros motivos en voluta. En el nivel superior, perteneciente al piso principal, se repite 
el mismo esquema de cinco vanos con arco de medio punto, todos ellos puertas que dan salida al balcón corrido con una barandilla muy simple de barras sin otra decoración. También aquí el arco poseía la misma rejería que en los inferiores. El ancho de los cinco vanos coincide con los del nivel inferior, por lo que la mayor anchura del central inferior se suple en este nivel con una también mayor anchura de los dos estribos centrales respecto a los restantes. De la misma manera en los estribos se alojan pilastras adosadas que, en el caso del arco central, se ven acompañadas de un casetón vertical decorado. Por encima se extendía una cornisa moldurada sobre la que apoyaba un antepecho o pretil macizo. Finalmente sobre éste se situaban seis jarrones de cerámica o piedra.

Con este último elemento entramos ya en el terreno de la ornamentación. Ésta se localizaba como se ha indicado en las enjutas con motivos vegetales típicamente renacentistas a candelieri con roleos y hojas de acanto, y en las pilastras, donde la decoración se concentra en un casetón con ovas enmarcadas por motivos geométricos. En el nivel superior las enjutas están recercadas por una moldura con un tipo de palmeta al interior. Finalmente, el antepecho posee una decoración de guirnaldas florales de gusto neoclásico.

Si comparamos el proyecto con el acabado final encontraremos una serie de diferencias únicamente ornamentales, pues a nivel compositivo se efectuaron los dos niveles de arcadas con pilastras adosadas en los estribos. En planta baja, debido a la conversión a mediados del siglo XX de los dos espacios a los lados del eje central en sendos locales comerciales (una heladería y una perfumería) alguno de los supuestos vanos con función de ventana enrejada se transformaron en puertas para dar servicio a los comercios. Asimismo la fachada en ambos casos se chapó de azulejos, cubriendo y ocultando las pilastras y las jambas de los arcos.

La ornamentación que finalmente se ejecutó puede ser calificada como de un mayor eclecticismo, pues sin abandonar el estilo neorrenacentista se introducen otros elementos neorromanos, de sabor más arqueologista, como es el caso de las acroteras, e incluso otros cercanos a la ornamentación barroca.

En las jambas de los arcos del nivel superior, en los capiteles de las pilastras, en las arquivoltas, en el friso corrido que cierra el tímpano de los arcos y en este último espacio se documentaron placas cerámicas o terracota bizcochada con relieves, material éste que se generalizó durante el periodo isabelino (fig. 9). En el caso de jambas, friso, arquivoltas y ábaco de los capiteles la decoración es siempre la misma, una faja dividida en dos molduras una en bocel con ovas y otra en cuarto de bocel con hojas de acanto contrapuestas. En el tímpano la decoración es de hojas de acanto entrelazadas, con una venera central y sobre ella un ave, supuestamente un águila, que se proyecta al exterior como una pieza de bulto redondo.

El tambor de los capiteles presenta un relieve compuesto de pájaros picoteando unas frutas, posiblemente uvas. Este es un motivo muy usual en la iconografía 
de finales del siglo XVII y la primera mitad del XVIII y por lo tanto de marcado carácter barroco e incluso rococó.

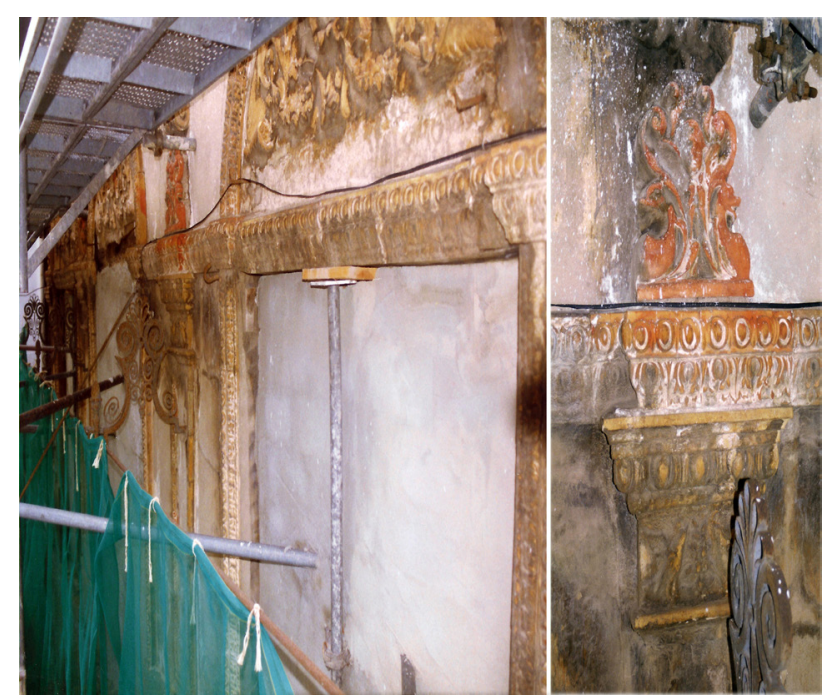

Fig. 9. Vistas de la decoración de placas de la fachada de la Casa de la Reina.

Por último, y frente al proyecto original, las enjutas se encuentran prácticamente desprovistas de decoración a excepción de una serie de plaquetas en forma de palmeta sobre los capiteles de las pilastras. Estas placas son de claro gusto clásico mostrando un putti flanqueado por animales fantásticos.

A pesar de que en la actualidad no se conservan, hasta hace pocos años, posiblemente hasta el momento del saqueo que se realizó en el edificio en el que fueron robados ciertos elementos decorativos del interior, existían sobre el antepecho (sustituyendo los jarrones del proyecto original), una serie de acroteras exentas en forma de palmeta que debían ser muy similares a las que hemos descrito sobre los capiteles de las pilastras del nivel superior.

Para finalizar con la descripción de la fachada indicaremos que el balcón posee una barandilla de barras de sección cuadrada con decoración de palmetas en la parte superior y unos elementos también en forma de palmeta que unen la barandilla con la fachada con pomos en forma de piña. Todavía se han conservado las barras móviles utilizadas para instalar un toldo.

\section{Estudio de la planta e interior de la vivienda}

La vivienda fue finalizada unos meses después que el taller, como reza la fecha ya de 1862 situada en la reja del tímpano de la puerta principal. La estructura general del edificio consiste en una serie de muros de carga transversales 
a la fachada, por un lado las medianeras Norte y Sur y por otro los dos muros que delimitan el pasaje o eje central de la vivienda con acceso desde la puerta principal. A partir de aquí se disponen una serie de crujías paralelas a la calle, en total cuatro, cuyos forjados quedan sustentados por pilares situados en las medianeras y en los muros de carga del eje, contando además con un patio al fondo del edificio (fig. 4).

El espacio central, a doble altura (planta baja y entresuelo), funciona como pasillo distribuidor de las estancias de las alas o manos laterales, registrándose un continuum de paso desde la calle hasta el patio, de manera que el tránsito de lo urbano a lo doméstico queda absolutamente matizado. No existe pues un cambio abrupto de ambientes, lo cual se refuerza por la ubicación en planta baja a lo largo de los dos muros de carga centrales de ventanas enrejadas iguales a los que se dispondrían en una fachada.

Se puede incluso calificar de escenario la manera en la que se dispone este eje central con los vanos laterales y la colocación de una columnata en la que tan sólo las dos columnas de hierro fundido del extremo Oeste tienen función portante, pues el resto se adosan a los muros de carga, relegándose a una función meramente decorativa.

En planta superior o principal todo el espacio es utilizado ya para habitaciones, si bien se mantiene la triple separación de ambientes en las dos alas laterales y la central, situándose en las laterales las habitaciones privadas y en la central los espacios semipúblicos, como son el comedor y el salón.

En planta baja la crujía central concentra la mayor parte de los elementos suntuarios de la casa y, aun vertebrando los accesos a las crujías laterales, se consigue un peculiar efecto de proyección al interior de un ambiente semiurbano (poco doméstico) gracias a la distribución en naves en profundidad con unos muros de carga donde vanos de reducida luz impiden la libre visión de los espacios laterales, junto con una serie de elementos singulares que seguidamente veremos.

Una vez pasado el umbral del gran arco principal se observa, en primer lugar, un espacio con una altura considerable, pues alcanza la planta baja y el entresuelo. En segundo lugar, la delimitación lateral que suponen los muros de carga focaliza la visión en profundidad como si se tratara de una calle, pero a la vez se es consciente de la existencia de dos grandes pantallas laterales ( interrumpidas sólo por vanos de luces reducidas), que refuerzan todavía más la impresión de que las alas presentan fachadas que dan a esa supuesta "calle", pues junto a esas puertas de paso a las habitaciones se colocan en un segundo nivel ventanas enrejadas que no difieren en modo alguno de las que se colocarían en los exteriores, diseñadas como falsos balcones, pues disponían de barandillas de hierro, como se desprende por las rozas dejadas en el paramento. 
Por último, el pavimento de esta entrada se efectúa con grandes losas de piedra, quedando la hilada de los extremos junto los muros laterales algo resaltada respecto a las centrales de manera que semejan aceras de una vía.

Como elementos suntuarios, el pasillo está jalonado por cuatro pares de columnas de fundición sobre un pedestal de piedra y coronadas por una figura en terracota (con varillaje interno metálico) de un ave, siete de las cuales fueron robadas (fig. 6B). Se según el sentir general se interpreta como un águila, aunque opinamos que más que un águila puede tratarse de una grulla o una garza por su largo cuello y patas, tratada con una cierta idealización que la convierte casi en un animal fantástico (fig. 6C).

A la altura del forjado toda la estancia está rodeada por un friso neorrenacentista que enlaza con los de la fachada, con los que tiene en común la cenefa de ovas superior. Se trata de placas de ladrillo cocido con decoración en relieve de tipo floral formando guirnaldas y que, como ocurría en los capiteles de las pilastras de la fachada, presentan parejas de pájaros picoteando frutas en el interior de la guirnalda. Finalmente, los florones del techo presentan la misma decoración que los frisos siguiendo una composición de círculos concéntricos. Parten de un rosetón central rodeado de un perlario, le sigue un segundo círculo de guirnaldas encadenadas de tema vegetal de hojas de acanto y un tercer círculo con el tema de los pájaros picoteando frutas enlazados por las mismas guirnaldas de hojas de acanto (fig. 10B).

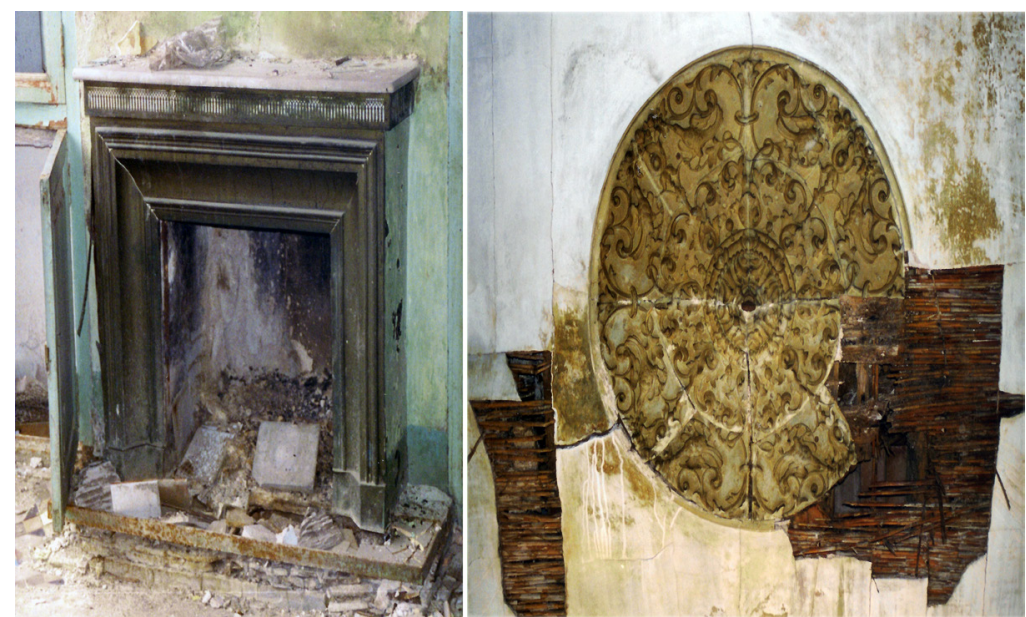

Fig. 10. A la izquierda, chimenea del salón. A la derecha, florón del techo de pasillo central de la planta baja.

Al entresuelo se accede por sendas escaleras independientes pues entre ambos laterales no existe comunicación al mediar el hueco del pasaje central que, 
como sabemos, ocupaba los dos primeros niveles. La escalera del ala izquierda únicamente da servicio al entresuelo, mientras que la del ala derecha sube al entresuelo, planta principal y desván. Este nivel acogió las pequeñas habitaciones del servicio, que se articulan por medio de un pasillo que atraviesa toda el ala, pegado al muro de carga del eje central. En este nivel se conservaba una muestra de pavimento de azulejos con decoración partida en diagonal, con una mitad en blanco y la otra con motivos florales en azul con fondo rayado. Estas piezas se combinan de manera que se forman chevrones y florones mediante la unión de cuatro piezas. Se encuadran en el estilo del eclecticismo de rayados (V.J. Estall: 2000, p. 181), que se datan en la década de 1860, como la vivienda (fig. 11B).

En la planta principal todo el espacio se hace habitable, incluida la nave central, la cual seguirá detentando la jerarquía espacial, pues es aquí donde se sitúan las dos estancias principales de carácter semipúblico, el comedor y el salón, frente a las habitaciones privadas de las alas. De nuevo será en estas dos habitaciones centrales donde encontraremos los elementos de mayor suntuosidad de la planta, como son, entre otros, la pavimentación a partir de baldosines tipo Nolla (Algarra y Camps: 1995, p. 126), pintura mural en las paredes del salón, tallas, chimenea y rejería de la balconada que da al patio.

El salón estaba pavimentado con baldosines de gres tipo Nolla ${ }^{13}$ de diferentes tamaños, formas y colores (fig. 11A). Poseía una cenefa que delimitaba la sala con motivos geométricos de losanges inclinadas, de piezas azules, blancas y marrones. La composición central formaba motivos en estrella, en los que se utilizaban piezas hexagonales, triangulares, cuadradas y romboides en azul, gris, blanco, rojo, negro y marrón. Las paredes estaban pintadas con motivos tales como un camafeo central azul, rodeado de motivos florales y sobre un zócalo con casetones marrones.

Desde el salón se accedía al comedor, nuevamente pavimentado con baldosines tipo Nolla, cuya composición consta de 8 tipos de baldosines: 1 hexagonal en gris, 4 triángulos equiláteros blancos, azules, rojos y negros, y 3 cuadrados blancos, marrones y grises. Con estas piezas se trazan series de octógonos a $45^{\circ}$ respecto a los muros, separados por hiladas de baldosines cuadrados y triangulares que forman octógonos más pequeños. Otros elementos documentados fueron una chimenea (fig.10A), heredera de las chimeneas neoclásicas, con un frente únicamente moldurado aparentemente de mármol de color. Sin embargo, poseía un remate metálico calado que recuerda a los motivos neogóticos con arcadas de arcos apuntados. Por ello se trataría de una chimenea que como el resto de la vivienda se encuadra en el romanticismo ecléctico. La rejería vuelve a ser un

13 En fechas recientes se ha celebrado en Meliana (Valencia) el I Congreso Nacional sobre la cerámica Nolla (9-10 de abril de 2015), https://congresonolla.wordpress.com/dirigido por los arquitectos Xavier Laumain y Ana López Sabater, donde se ha tratado en profundidad este singular pavimento de gres producido en la fábrica Nolla, ubicada en 1860 en plena huerta de Valencia en la localidad de Melina. 
elemento singular en la ambientación de esta sala. El cierre del salón en la parte recayente al patio estaba resuelto mediante una gran reja que se extendía de parte a parte sin existir ninguna porción de muro, por lo que estructuralmente este paño estaba en su totalidad abierto, decorada con pletinas que formaban motivos geométricos de hexágonos y rombos y un friso de volutas. La rejería daba paso a una terraza que se extendía a lo largo de todo el frente de las tres naves (la central del salón más las dos laterales).

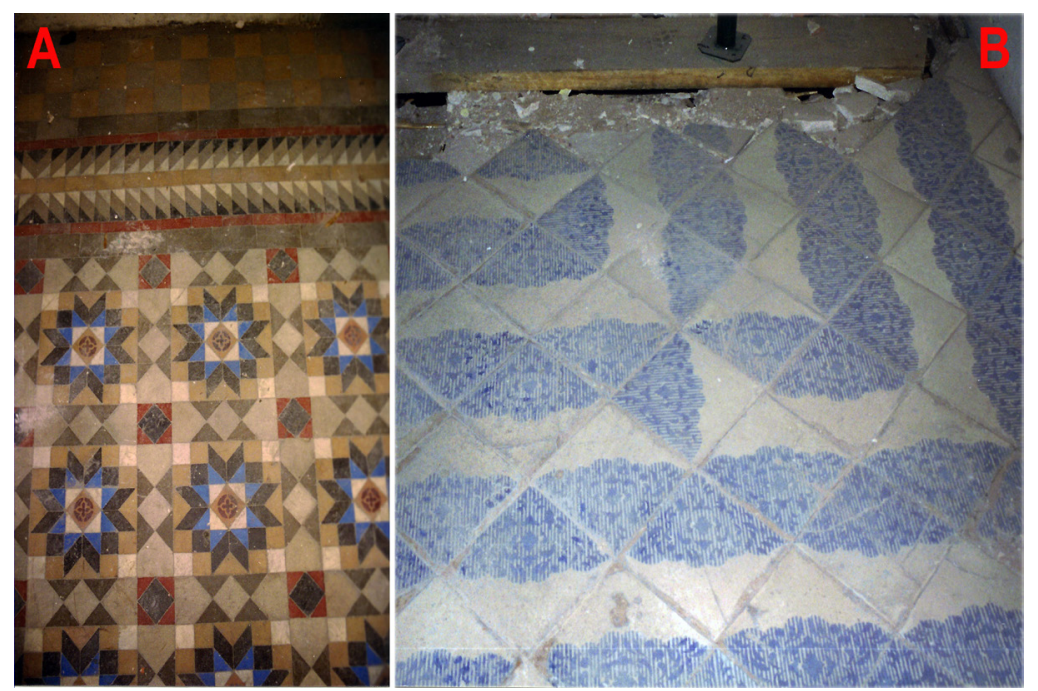

Fig. 11. A. Pavimento de baldosines de gres tipo Nolla del salón. B. Pavimento de azulejos del entresuelo del tipo eclecticismo de rayados (1860).

\section{Conclusión}

El estudio archivístico y el análisis arqueológico permitieron reconstruir la historia arquitectónica y de la propiedad del edificio de la calle de la Reina 85 en el Cabañal de Valencia, proporcionando de esta manera la información suficiente para valorar en su justa medida la importancia patrimonial del inmueble y de los elementos que lo integraban como paso previo e imprescindible (o al menos así debería ser) a la rehabilitación de este edificios históricos. La vivienda en cuestión se inscribe en un contexto urbano que tiene su origen en la planificación urbanística realizada por la Bailía General de Valencia y el ayuntamiento de Pueblo Nuevo del Mar entre los años 1839 y 1840, la cual da como resultado la parcelación de las manzanas de casas comprendidas entre las actuales calle de Francisco Cubells y la avenida del Mediterráneo con eje en la calle de la Reina.

Las motivaciones de esta planificación explican claramente el por qué de la situación en este punto de la ciudad de una vivienda con las características arqui- 
tectónicas que ya conocemos. Se trata de un barrio que nació como respuesta al deseo de la burguesía del periodo isabelino de poseer una residencia de recreo durante los meses de verano para tomar los apreciados baños de mar. Comprenderemos así cómo a lo largo de esta calle vamos a encontrar residencias de alto valor arquitectónico y patrimonial, en la que existían además edificios de cierta envergadura como el Teatro de la Marina hoy ya derribado.

A la sombra de estos grandes edificios, que copaban las alineaciones más próximas a la playa, se fueron construyendo por los mismo años y en décadas posteriores otras viviendas en las alineaciones traseras a éstas y al norte del límite de la primera urbanización, constituido por la acequia del Gas (actual avda. del Mediterráneo), siguiendo la organización ortogonal primitiva. Estos edificios, unas veces casas y otras barracas acogieron a las clases más populares, en muchos casos los antiguos pescadores del Cabañal, pero también agricultores de la próxima huerta.

El resultado fue una trama urbana hoy calificada de Bien de Interés Cultural, de la que desde hace ya demasiados años pende una espada de Damocles ante el proyectado derribo parcial para proyectar la Avda. de Blasco Ibáñez hasta la misma playa (Plataforma Salvem el Cabanyal). Una ruptura innecesaria por cuanto el acceso al mar está plenamente asegurado, más si cabe si reconocemos el valor urbano del Cabañal a través de los valores arquitectónicos, que como la Casa del a Reina, lo forman.

\section{Bibliografía}

V.M. ALGARRA PARDO: Memoria histórico-arqueológica de la vivienda de la c/ de la Reina, 85. El Cabanyal, Valencia. Informe inédito Conselleria de Cultura. Dirección General de Patrimonio Cultural Valenciano. 1999.

V.M. ALGARRA PARDO y C. CAMPS: "Baldosa". En M. CERDÀ y M. GARCÍA BONAFÉ (dir.). Enciclopedia Valenciana de Arqueología Industrial. Diputación Provincial de Valencia. Valencia, p. 125-126. 1995.

V.J. ESTALL I POLES: Catálogo de la colección de azulejos de serie del siglo XIX. Museo del azulejo de Onda. Faenza editrie ibérica, s.l. 2000.

R. PASTOR VILLA: El Cabanyal: Lectura de las estructuras de la edificación. Ensayo tipológico residencial. 1900-1936. Tesis Doctoral. Universidad Politécnica de Valencia. 2012.

A. SANCHIS PALLARÉS: Historia del Cabanyal. Poble Nou de la Mar (1238-1897). Javier Boronat, editor, Valencia: 1997.

R. SOLAZ: "Dombón, un pionero de la aviación española. Vecino de la calle de la Reina, en el Cabanyal". Valencia noticias, 21 de marzo de 2014, http:// vlcnoticias.com/dombon-un-pionero-de-la-aviacion-espanola-vecino-de-lacalle-de-la-reina-en-el-cabanyal/. 